\title{
Sintomas Relacionados à Diminuição de Ingestão Alimentar em Pacientes com Neoplasia do Aparelho Digestório Atendidos por um Programa de Internação Domiciliar
} Symptoms Related to Decreased Food Intake in Patients with Cancer of the Digestive System Served by a Home Care Program Síntomas Relacionados a la Disminución de la Ingesta Alimentar en Pacientes con Neoplasia del Aparato Digestivo atendidos por un Programa de Internación Domiciliaria

Évelyn de Sousa Araújo ${ }^{1}$; Patrícia Abrantes Duval ${ }^{2}$; Denise Halpern Silveira ${ }^{3}$

\section{Resumo}

Introdução: Entre os fatores associados à desnutrição no câncer, os sintomas relacionados à ingestão alimentar podem contribuir de forma significativa para o comprometimento do estado nutricional. Objetivo: Verificar a prevalência de sintomas relacionados à diminuição da ingestão alimentar e sua associação com a localização do tumor, de pacientes portadores de neoplasia do aparelho digestório, internados num Programa de Internação Domiciliar Interdisciplinar Oncológico. Método: Estudo descritivo que utilizou dados secundários de um Programa de Internação Domiciliar Interdisciplinar Oncológico. Participaram do estudo todos os pacientes, atendidos pela Nutrição, com diagnóstico de neoplasia do aparelho digestório internados no programa no período de fevereiro de 2006 a fevereiro de 2011 e foram analisados seus dados demográficos, clínicos e nutricionais. Resultados: Dos 102 pacientes analisados, com idade média de 61+14,1 anos, 100\% foram considerados com algum grau de desnutrição. O câncer de intestino foi o mais predominante em ambos os sexos, seguido do câncer de esôfago. Os sintomas mais prevalentes corresponderam à xerostomia $(75,5 \%)$, saciedade precoce $(74,5 \%)$, dor $(60,8 \%)$ e anorexia $(53,9)$. A prevalência de disfagia nos pacientes com tumor de esôfago foi aproximadamente quatro vezes maior quando comparados aos pacientes com outros tumores do aparelho digestório ( $\mathrm{p}<0,01$ IC 95\% 2,35-6,48). Conclusão: A xerostomia, saciedade precoce, dor e anorexia podem ter contribuído para menor ingestão alimentar, o que agrava o estado nutricional desses pacientes. Através desse conhecimento, estratégias nutricionais poderão ser desenvolvidas a fim de prevenir e ou melhorar essa condição. Palavras-chave: Neoplasias do Sistema Digestório; Ingestão de Alimentos; Desnutrição; Sintomas; Estudos Transversais; Epidemiologia Descritiva

\footnotetext{
Trabalho realizado no Programa de Internaçáo Domiciliar Interdisciplinar do Hospital Escola (HE) da Universidade Federal de Pelotas (UFPel). Pelotas (RS), Brasil.

${ }^{1}$ Nutricionista. Especialista em Saúde da Família e Residente do Programa de Pós-Graduação Residência Integrada Multiprofissional em Saúde. Área de Concentração Atenção à Saúde Oncológica. HE/FAU/UFPel. Pelotas (RS), Brasil. E-mail: evelyndsousa@yahoo.com.br.

${ }^{2}$ Nutricionista. Mestre em Nutriçáo e Alimentos. Nutricionista do HE da UFPel. Pelotas (RS), Brasil. E-mail: patricia-duval@hotmail.com.

${ }^{3}$ Nutricionista. Doutoranda do Programa de Pós-Graduação em Ciências da Saúde da Fundação Universidade do Rio Grande. Rio Grande (RS), Brasil. Professora Adjunta do Departamento de Nutrição da UFPel. Pelotas (RS), Brasil. E-mail: denise_hs@hotmail.com.

Endereço para correspondência: Évelyn de Sousa Araújo. Rua Anécio de Carvalho, 200. São Jose. Arroio Grande (RS), Brasil. CEP: $96330-000$.
} 


\section{INTRODUÇÃO}

O câncer é considerado a principal causa de morte em países desenvolvidos, ficando em segundo lugar nos países em desenvolvimento. Segundo recente relatório da Agência Internacional para Pesquisa em Câncer (IARC) / Organização Mundial da Saúde (OMS), o impacto global do câncer dobrou em 30 anos $^{1,2}$.

Entre as neoplasias do aparelho digestório mais incidentes no Brasil, destacam-se a de estômago, cólon, reto, cavidade oral e esôfago, em ambos os sexos. A Região Sul apresenta níveis mais elevados de mortalidade entre pacientes portadores de neoplasias do aparelho digestório, quando comparada com outras regiốes ${ }^{2}$.

Os pacientes com câncer cursam com inúmeros problemas, sendo a desnutrição um dos mais prevalentes, podendo ser encontrada entre $40 \%$ e $80 \%$ dos pacientes no momento do diagnóstico. A desnutrição no paciente com câncer é multifatorial, dependente da localização do tumor, terapêutica empregada (quimioterapia, radioterapia e cirurgia), e estadiamento da doença ${ }^{3,4}$.

Entre os fatores associados à desnutrição, os sintomas relacionados à ingestão alimentar, como: náuseas, vômitos, diarreia, saciedade precoce, obstipação intestinal, xerostomia, disgeusia e disfagia, podem contribuir de forma significativa para o comprometimento do estado nutricional ${ }^{5,6}$.

Pacientes portadores de câncer do aparelho digestório, além do estresse metabólico imposto pelo tumor, apresentam alteraçóes mecânicas e fisiológicas que podem interferir na ingestão, digestão e a absorção adequada dos alimentos, levando a um inadequado aproveitamento de nutrientes?.

A avaliação do estado nutricional no paciente oncológico é considerada de grande importância, tendo em vista que o déficit nutricional está associado a maiores índices de morbidade e mortalidade, infecçáo, maior tempo de hospitalização, menor resposta à quimioterapia e radioterapia e maior custo hospitalar ${ }^{8}$.

Dos instrumentos propostos para avaliação nutricional de pacientes oncológicos, destaca-se a Avaliaçáo Subjetiva Global Produzida pelo Paciente (ASG-PPP), em versão traduzida e adaptada para o português por Gonzalez et al. ${ }^{7,9,10}$.

Durante o tratamento antineoplásico, os pacientes que não têm indicação de hospitalização, mas necessitam de assistência semelhante à oferecida em ambiente hospitalar, se beneficiam da internação domiciliar ${ }^{11}$.

A internação domiciliar proporciona a prestação de cuidados sistematizados de forma integral e contínua no domicílio, com supervisão e ação da equipe de saúde específica, personalizada, centrada na realidade do paciente e envolvendo a família, podendo ou não utilizar equipamentos e materiais ${ }^{12}$.
Sendo assim, diante da problemática que envolve o paciente com câncer, principalmente os de aparelho digestório, torna-se importante a identificação precoce dos sintomas relacionados à ingestão alimentar para que a intervenção nutricional, juntamente com atuaçáo da equipe multidisciplinar, possa auxiliar na tolerância ao tratamento, alívio dos sintomas e manutenção do estado nutricional como forma de proporcionar uma melhor qualidade de vida.

O objetivo deste estudo foi verificar a prevalência de sintomas relacionados à diminuição da ingestão alimentar e sua associaçáo com a localização do tumor, de pacientes portadores de neoplasia do aparelho digestório internados num Programa de Internação Domiciliar Interdisciplinar (PIDI) Oncológico.

\section{MÉTODO}

Foi realizado um estudo descritivo usando dados secundários de um PIDI Oncológico. Foram incluídos no estudo todos os pacientes atendidos pela nutrição com diagnóstico de tumores digestivos no período de fevereiro de 2006 a fevereiro de 2011, não havendo nenhuma exclusão.

O PIDI Oncológico é formado por uma equipe interdisciplinar composta por um médico assistencial, enfermeiro, técnico de enfermagem, nutricionista, assistente social, psicólogo, assistente espiritual, acadêmicos de nutrição e enfermagem ${ }^{13}$. No ano de 2010, os alunos da Residência Integrada Multiprofissional em Saúde - Área de Concentração Atenção à Saúde Oncológica do Hospital Escola/Fundação Apoio Universitário/Universidade Federal de Pelotas passaram a integrar a equipe do PIDI Oncológico, sendo assim acrescentados à equipe mais um profissional da área de nutrição, de psicologia, de enfermagem e de odontologia.

$\mathrm{O}$ atendimento nutricional aos pacientes internados no programa, ocorre através de visitas semanais feitas por um nutricionista, um residente de nutrição e um estagiário da Faculdade de Nutrição da UFPel.

A assistência nutricional aos pacientes inclui o preenchimento de um formulário com dados de identificação, avaliação antropométrica, anamnese alimentar e avaliação do estado nutricional através da ASG-PPP?

A ASG-PPP diferencia-se dos demais métodos de avaliação nutricional utilizados na prática clínica por englobar não apenas alteraçóes da composição corporal, mas também alteraçôes funcionais do paciente, além de identificar a ocorrência de complicaçóes associadas ao estado nutricional, como a presença de sintomas que interfiram na ingestáo alimentar ${ }^{7}$.

A ASG-PPP fornece uma classificação categórica (ASG A: bem nutrido ou anabólico, ASG B: desnutrição 
moderada ou suspeita, ASG C: gravemente desnutrido) além de uma escala numérica, na qual valores iguais ou superiores a quatro indicam risco nutricional e necessidade de intervenção nutricional ${ }^{7,9,10}$.

Foram analisados os dados referentes ao sexo, idade, localização do tumor, administração de terapias antineoplásicas, estadiamento, alteraçôes na ingestão alimentar, presença de sintomas, além das classificaçôes da ASG-PPP.

A alteração da ingestão alimentar foi considerada quando o paciente referia uma diminuição da ingestão no mês anterior à visita domiciliar ou alteraçóes relacionadas à consistência e/ou quantidade da dieta tolerada atualmente.

Os sintomas analisados foram os referidos durante as duas últimas semanas que antecederam à aplicação da ASG-PPP, impedindo que o paciente se alimentasse normalmente, sendo eles: anorexia, náusea, vômito, constipação, diarreia, mucosite, xerostomia, disgeusia, disosmia, disfagia, saciedade precoce ou dor ${ }^{7,10,14}$.

Os dados coletados foram digitados em planilha do software Microsoft Excel ${ }^{R}$ e exportados e analisados no programa $\operatorname{ttata}^{R}$, versão 11.2. Inicialmente procedeu-se à descrição das características dos pacientes estudados.

Foram realizadas análises bivariadas através de testes qui-quadrados e regressão de Poisson com variância robusta, devido à alta prevalência do desfecho. O nível de significância de $5 \%$ foi considerado para todas as análises.

O presente estudo foi aprovado pela Comissão de Educação e Assessoria à Pesquisa do Hospital Escola/ UFPel e, posteriormente, pelo Comitê de Ética em Pesquisa da Faculdade de Medicina da UFPel (Of. 31/11).

\section{RESULTADOS}

Foram, avaliados, pela equipe da Nutrição do PIDI Oncológico, 102 pacientes portadores de neoplasia do aparelho digestório internados no programa no período de fevereiro de 2006 a fevereiro de 2011.

As características demográficas, clínicas e nutricionais dos pacientes estão apresentadas na Tabela 1 .

A amostra apresentou-se homogênea com relação ao gênero, sendo a média de idade de 61 anos $\pm 14,1$. Com relação à avaliação do estado nutricional, $42 \%$ $(\mathrm{n}=42)$ dos pacientes encontravam-se com déficit nutricional segundo o índice massa corporal (IMC $<18,5$ $\mathrm{KG} / \mathrm{m} 2$ ), enquanto 38,7 (36) e $61,3 \%$ (57) foram considerados moderadamente e gravemente desnutridos pela classificação categórica da ASG-PPP, respectivamente.

Com relação à classificação numérica da ASG-PPP, observou-se que 99\% ( $\mathrm{n}=101)$ dos pacientes obtiveram um escore maior de nove pontos, indicando a necessidade crítica de intervençáo nutricional.

Nas características clínicas da amostra, 88,3\% ( $\mathrm{n}=89)$ dos indivíduos encontravam-se no estadiamento graus III e IV da doença, e apenas 12,8\% ( $\mathrm{n}=13)$ do total da amostra não apresentava metástases (Tabela 1 ).

O câncer de intestino foi o mais prevalente em ambos os sexos, acometendo $36,3 \%(\mathrm{n}=37)$ dos pacientes, seguido do câncer de esôfago, representando $24,5 \%$ $(\mathrm{n}=25)$ da amostra (Tabela 1$)$.

Quanto ao tratamento antineoplásico, 55,9\% ( $\mathrm{n}=57)$ realizaram algum tipo de cirurgia, $46,1 \%(n=47)$ e $9,8 \%$ $(n=10)$ dos pacientes encontravam-se em quimioterapia e radioterapia, respectivamente (Tabela 1 ).

A relação entre o tipo de tumor e a classificação categórica da ASG-PPP (A, B ou C) demonstrou que pacientes com câncer de estômago, esôfago e pâncreas apresentavam-se gravemente desnutridos no momento da entrevista.

O câncer de esôfago foi o mais prevalente entre os homens $35 \%$ ( $\mathrm{n}=18)$ e, entre as mulheres, o de intestino $45 \%(n=23)$, com diferença significativa entre os sexos.

Foi observado que a ingestão alimentar no último mês esteve diminuída significativamente nos pacientes que apresentaram náuseas $70,5 \%(\mathrm{n}=31)$ e disgeusia $76,7 \%$ $(\mathrm{n}=33)(\mathrm{p}<0,05)$.

$\mathrm{Na}$ ingestão alimentar atual, $78 \%(\mathrm{n}=29)$ dos pacientes com câncer de intestino e $71 \%(\mathrm{n}=10)$ com câncer de pâncreas apresentavam uma diminuição significativa no momento da primeira visita $(\mathrm{p}<0,05)$. Sendo que $50 \%$ $(\mathrm{n}=17)$ dos pacientes que recebiam alimentação por sondas enterais apresentavam disfagia como sintoma mais prevalente $(\mathrm{p}<0,001)$.

A alteraçáo do peso nos últimos 15 dias que precedeu à entrevista revelou que $75 \%(\mathrm{n}=54)$ dos pacientes com xerostomia apresentaram perda de peso significativa $(\mathrm{p}=0,02)$.

Os sintomas com uma prevalência maior de $50 \%$ foram a xerostomia, saciedade, dor e anorexia (Tabela 2).

A prevalência de disfagia nos pacientes com tumor de esôfago foi quatro vezes maior quando comparados aos pacientes com outros tumores do aparelho digestório ( $\mathrm{p}<0,01$ RR 3,9 IC 95\% 2,35-6,48) (Tabela 3).

\section{DISCUSSÃO}

Neste estudo, todos os pacientes encontravam-se com algum grau de desnutrição segundo a ASG-PPP, entre eles, $61 \%(n=57)$ foram classificados como desnutridos graves. Quando realizada através do IMC, observou-se que somente $42,0 \%(\mathrm{n}=42)$ dos pacientes foram classificados com déficit nutricional ${ }^{15}$.

A ASG-PPP apresentou alta sensibilidade e especificidade na identificação de pacientes desnutridos com diagnóstico de câncer. No entanto, o IMC subestimou o percentual de pacientes desnutridos. Uma explicação para a ineficiência do IMC em detectar pacientes oncológicos com desnutriçáo se deve ao fato de não considerar a composição corporal ${ }^{13}$. 
Tabela 1. Caracterização dos pacientes com neoplasias do aparelho digestório internados no PIDI Oncológico de fevereiro, de 2006 a fevereiro de 2011. Pelotas (RS) ( $n=102)$

\begin{tabular}{|c|c|c|}
\hline Variáveis & $\mathbf{n}$ & $\%$ \\
\hline \multicolumn{3}{|l|}{ Sexo } \\
\hline Masculino & 51 & 50,0 \\
\hline Feminino & 51 & 50,0 \\
\hline \multicolumn{3}{|l|}{ Idade } \\
\hline 30 a 40 anos & 8 & 7,8 \\
\hline 41 a 50 anos & 21 & 20,6 \\
\hline 51 a 59 anos & 22 & 21,6 \\
\hline Maior de 60 anos & 51 & 50,0 \\
\hline \multicolumn{3}{|l|}{ IMC } \\
\hline Déficit de peso & 42 & 42,0 \\
\hline Peso adequado & 41 & 41,0 \\
\hline Sobrepeso & 14 & 14,0 \\
\hline Obesidade & 03 & 3,0 \\
\hline \multicolumn{3}{|l|}{ Estado nutricional (classificação categórica da ASG-PPP) } \\
\hline Bem nutrido $(A)$ & - & - \\
\hline Moderadamente desnutrido ou suspeita de desnutrição (B) & 36 & 38,7 \\
\hline Gravemente desnutrido (C) & 57 & 61,3 \\
\hline \multicolumn{3}{|l|}{ Classificação numérica da ASG-PPP } \\
\hline 0-1: Não há necessidade de intervenção & - & - \\
\hline 2-3: Educação do paciente e seus familiares & - & - \\
\hline 4-8: Necessita intervenção nutricional & 1 & 1,0 \\
\hline$\geq 9$ : Necessidade crítica de intervenção nutricional e manejo dos sintomas & 101 & 99,0 \\
\hline \multicolumn{3}{|l|}{ Características clínicas } \\
\hline \multicolumn{3}{|l|}{ Local do tumor primário } \\
\hline Intestino & 37 & 36,3 \\
\hline Esôfago & 25 & 24,5 \\
\hline Estômago & 19 & 18,6 \\
\hline Pâncreas & 14 & 13,7 \\
\hline Fígado e vias biliares & 7 & 6,9 \\
\hline Presença de metástase & 89 & 87 \\
\hline \multicolumn{3}{|l|}{ Estadiamento } \\
\hline I & - & - \\
\hline II & 12 & 11,7 \\
\hline III & 22 & 21,6 \\
\hline IV & 68 & 66,7 \\
\hline \multicolumn{3}{|l|}{ Tipos de tratamento } \\
\hline Quimioterapia durante a internação no PIDI Oncológico & 47 & 46,1 \\
\hline Radioterapia durante a internação no PIDI Oncológico & 10 & 9,8 \\
\hline Cirurgia & 57 & 55,9 \\
\hline
\end{tabular}

A importância de se detectar precocemente o déficit nutricional está estreitamente relacionada com diminuição da resposta ao tratamento oncológico e à qualidade de vida, com maiores riscos de complicaçôes pós-operatórias, aumento na morbimortalidade, aumento do tempo de internação e no custo hospitalar ${ }^{16}$.

Ravasco ${ }^{17}$, em um estudo sobre a deterioraçáo do estado nutricional em pacientes com câncer de cabeça e pescoço, cólon e reto, e do aparelho digestório com estadiamentos III e IV, também encontrou resultado semelhante, em que a
ASG-PPP demonstrou alta sensibilidade e especificidade, e forte capacidade de detectar pacientes em risco nutricional, em comparação ao IMC.

Segundo Waitzberg ${ }^{7}$, os cânceres do trato digestório, justamente por estarem localizados em órgão responsáveis pela nutrição (ingestão, absorção e utilização de nutrientes), são frequentemente associados à incidência de desnutrição. $\mathrm{O}$ presente estudo reforça esse dado, tendo em vista que todos os pacientes avaliados apresentaram algum grau de desnutrição já no momento da primeira avaliação. 
Tabela 2. Prevalência de sintomas em pacientes portadores de neoplasia do aparelho digestório internados no PIDI Oncológico, 2006-2011

\begin{tabular}{l|c|c}
\multicolumn{1}{c|}{ Sintomas } & $\mathbf{n}$ & $\%$ \\
\hline Xerostomia & 77 & 75,5 \\
\hline Saciedade & 76 & 74,5 \\
\hline Dor & 62 & 60,8 \\
\hline Anorexia & 55 & 53,9 \\
\hline Constipação & 47 & 46,1 \\
\hline Disosmia & 47 & 46,1 \\
\hline Náuseas & 44 & 43,1 \\
\hline Disgeusia & 43 & 42,2 \\
\hline Vômitos & 35 & 34,3 \\
\hline Disfagia & 34 & 33,3 \\
\hline Diarreia & 12 & 11,8 \\
\hline Mucosite & 10 & 9,8 \\
\hline
\end{tabular}

Quanto ao escore numérico da ASG-PPP, observou-se que a grande maioria dos pacientes estudados obteve um escore maior de nove pontos, indicando a necessidade de intervenção nutricional. Andrew et al. ${ }^{18}$, em um estudo de coorte, avaliaram 79 pacientes com câncer avançado e em cuidados paliativos, utilizando duas ferramentas de avaliação para quantificar o impacto dos sintomas associados ao câncer induzido pela síndrome de anorexia caquexia, sendo uma delas a ASG-PPP. Neste estudo, todos os pacientes acompanhados apresentaram escore numérico maior que nove pontos, indicando necessidade crítica de intervenção nutricional, o que corrobora os achados do presente trabalho.
Com relação à localização do tumor, foi evidenciada uma maior prevalência de tumor de intestino e esôfago em ambos os sexos. Segundo estimativas do Instituto Nacional de Câncer José Alencar Gomes da Silva (INCA), válidas para os anos de 2012 e 2013, na regiáo Sul, o câncer de colón e reto será o terceiro mais frequente nos homens e o segundo nas mulheres. Já o câncer de esôfago será o quinto mais frequente em homens, ocupando a posição de décimo primeiro nas mulheres ${ }^{2}$.

No presente estudo, $57 \%$ dos pacientes estavam ou já haviam realizado tratamento quimioterápico e/ou radioterápico no momento da avaliação; e, segundo o Consenso Nacional de Nutriçáo Oncológica do INCA, o tratamento antineoplásico influencia negativamente no estado nutricional dos pacientes com câncer ${ }^{16}$.

Pacientes em tratamento antineoplásico apresentam vários sinais e sintomas que levam à diminuição da ingestão diária de nutrientes o que compromete o estado nutricional. Durante o tratamento, os pacientes oncológicos podem evoluir para desnutrição moderada ou grave e cerca de $20 \%$ desses pacientes morrem em decorrência da desnutrição e não da doença maligna ${ }^{19}$.

Marin $^{20}$, ao avaliar a evoluçáo do risco nutricional de acordo com a terapia antineoplásica aplicada em 226 pacientes oncológicos, também verificou alta prevalência de desnutriçáo; cerca de $60 \%$ dos pacientes com câncer estavam desnutridos. Essa prevalência aumentou para $81 \%$ naqueles que estavam em cuidados paliativos. Aproximadamente $70 \%$ apresentaram alguma dificuldade para se alimentar. Mais de a metade dos pacientes necessitaram de aconselhamento nutricional e controle dos sintomas que interferem na ingestão de alimentos.

Tabela 3. Prevalência de sintomas segundo a localização do tumor em pacientes portadores de neoplasia do aparelho digestório internados no PIDI Oncológico, 2006-2011

\begin{tabular}{l|c|c|c|c|c|c|}
\hline \multirow{2}{*}{ Sintoma } & \multicolumn{7}{c}{ Local fumor } \\
\cline { 2 - 7 } & $\begin{array}{c}\text { Esôfago } \\
\mathbf{n}(\%)\end{array}$ & $\begin{array}{c}\text { Gástrico } \\
\mathbf{n}(\%)\end{array}$ & $\begin{array}{c}\text { Intestino } \\
\mathbf{n}(\%)\end{array}$ & $\begin{array}{c}\text { Pâncreas } \\
\mathbf{n}(\%)\end{array}$ & $\begin{array}{c}\text { Fígado/via } \\
\text { biliares n (\%) }\end{array}$ & p* \\
\hline Anorexia & $13(52,0)$ & $9(47,4)$ & $24(64,9)$ & $6(42,9)$ & $3(42,9)$ & 0,53 \\
\hline Náusea & $9(36,0)$ & $9(47,4)$ & $18(48,6)$ & $5(35,7)$ & $3(42,9)$ & 0,84 \\
\hline Vômito & $8(32,0)$ & $7(36,8)$ & $13(35,1)$ & $4(28,6)$ & $3(42,9)$ & 0,97 \\
\hline Constipação & $11(44,0)$ & $6(31,6)$ & $20(54,1)$ & $7(50,0)$ & $3(42,9)$ & 0,61 \\
\hline Diarreia & $3(12,0)$ & $2(10,5)$ & $7(18,9)$ & 0 & 0 & 0,44 \\
\hline Mucosite & $3(12,0)$ & 0 & $6(16,2)$ & 0 & $1(14,3)$ & 0,20 \\
\hline Xerostomia & $20(80,0)$ & $11(57,9)$ & $32(86,5)$ & $9(64,3)$ & $5(71,4)$ & 0,12 \\
\hline Disgeusia & $10(40,0)$ & $6(31,6)$ & $18(48,7)$ & $5(35,7)$ & $4(57,1)$ & 0,67 \\
\hline Disosmia & $12(48,0)$ & $9(47,4)$ & $15(40,5)$ & $7(50,0)$ & $4(57,1)$ & 0,93 \\
\hline Disfagia & $19(76,0)$ & $8(42,1)$ & $4(10,8)$ & $2(14,3)$ & $1(14,3)$ & $<0,001$ \\
\hline Saciedade & $18(72,0)$ & $17(89,5)$ & $25(67,6)$ & $11(78,6)$ & $5(71,4)$ & 0,48 \\
\hline Dor & $16(64,0)$ & $9(47,4)$ & $26(70,3)$ & $9(64,3)$ & $2(28,6)$ & 0,21 \\
\hline
\end{tabular}

*Teste exato de Fisher 
No presente estudo, segundo a ASG-PPP, a xerostomia, a saciedade precoce foram os sintomas mais prevalentes. Em estudo de revisão, Davis et al. citam, em análise prospectiva, com 1.000 pacientes da Unidade de Medicina Paliativa da Cleveland Clinic, que a saciedade precoce foi encontrada entre os dez sintomas mais prevalentes. Muitos fatores podem estar envolvidos na origem da saciedade precoce, como centro sensorial específico da saciedade, aversôes alimentares, mudanças diurnas na ingestão, motilidade e acomodação gástrica e hormônios gastrointestinais ${ }^{21}$.

A disfagia foi o sintoma que prevaleceu nos pacientes com câncer de esôfago quando comparado com outros tumores do aparelho digestório. Esse resultado também foi encontrado por Monteiro ${ }^{22}$, em um estudo com pacientes com câncer de esôfago em estádio avançado da doença.

As altas prevalências evidenciadas entre os pacientes do PIDI, para ambos os sintomas, podem ser influenciadas por alguns fatores que caracterizam essa população, como a presença de doença metastática em $87 \%$ dos indivíduos, além de eles apresentarem idade acima de 60 anos. Sabe-se que o estadiamento avançado da doença e a presença de metástases são fatores determinantes para o mau prognóstico do estado nutricional desses pacientes, influenciando diretamente na perda ponderal e de apetite ${ }^{23}$.

Segundo estudo de Silva et al. ${ }^{24}$, uma açáo conjunta de uma equipe de saúde, como a que ocorre no PIDI Oncológico, pode contribuir diretamente para o controle dos sintomas, permitindo a discussão quanto à conduta individualizada, promovendo o controle adequado da dor, aumento da ingestão alimentar e auxiliando o paciente oncológico a viver com melhor qualidade de vida.

\section{CONCLUSÃO}

Através deste estudo, foi possível conhecer o perfil dos pacientes portadores de neoplasia do aparelho digestório, atendidos durante cinco anos do PIDI oncológico: pacientes com idade acima de 60 anos, portadores de doença em estádio avançado, com alta prevalência de desnutriçáo e de sintomas associados ao tratamento antineoplásico.

Os sintomas mais prevalentes foram xerostomia, saciedade precoce, dor e anorexia os quais, somados à sintomatologia imposta pelo tratamento e à presença física do tumor no trato digestório, podem ter contribuído para menor ingestáo alimentar, o que agravou o estado nutricional desses pacientes. Através desse conhecimento, estratégias nutricionais poderão ser desenvolvidas a fim de prevenir e ou melhorar essa condição.

\section{CONTRIBUIÇÕES}

Évelyn de Sousa Araújo, Patrícia Abrantes Duval e Denise Halpern Silveira contribuíram na concepção e planejamento do projeto de pesquisa; na obtenção e/ou análise e interpretação dos dados; na redação e revisão crítica.

\section{Declaraçáo de conflito de interesses: Nada a declarar}

\section{REFERÊNCIAS}

1. World Health Organization. World Cancer Report, 2008. International Agency for Research on Cancer, Lyon. 2009.

2. Instituto Nacional de Câncer José Alencar Gomes da Silva. Estimativa 2012: incidência de câncer no Brasil. Rio de Janeiro: INCA; 2011.118 p.

3. Correa PH, Shibuya E. Administração da terapia nutricional em cuidados paliativos. Revista brasileira de cancerologia 2007; 53(3): 317-23.

4. Yavuzsen T, Walsh D, Davis MP, Kirkova J, Jin T, Legrand $S$, et al. Components of the anorexia-cachexia syndrome: gastrointestinal symptom correlates of cancer anorexia. Support Care Cancer 2009; 17: 1531-41.

5. Fonseca DAF, Garcia RRM, Stracieri APM. Perfil nutricional de pacientes portadores de neoplasias segundo diferentes indicadores. Nutrir Gerais 2009; 3 (5): 444-61.

6. Duval PA, Vargas BL, Fripp JC, Arrieira ICO, Lazzeri B, Destri K, et al. Caquexia em Pacientes Oncológicos Internados em um Programa de Internação Domiciliar Interdisciplinar. Revista brasileira de cancerologia 2010; 56 (2): 207-12.

7. Waitzberg DL. Dieta, Nutrição e Câncer. São Paulo: Atheneu; 2006. 783p.

8. Silva MPN. Síndrome Anorexia/Caquexia em Câncer: Abordagem Terapêutica. Revista brasileira de cancerologia 2006; 52(1): 59-77.

9. Silva MCGB, Barros AJD. Avaliação nutricional subjetiva. Parte 2- Revisão de suas Adaptações e utilizações nas diversas especialidades clínicas. Arquivo Gastroenterologia 2002; 39(4): 248-52.

10. Mota MA, Andrade LL de, El-Kik RM, D'Ambrosi G. Avaliação subjetiva global e avaliação subjetiva global produzida pelo paciente em oncologia: um estudo comparativo. Revista brasileira de nutrição clínica 2009; 24(3): 196-202.

11. Brasil. Portaria No 2.529 de 19 de outubro de 2006. Institui a Internação Domiciliar no âmbito do Sistema Único de Saúde. Brasília (DF): Diário Oficial da República Federativa do Brasil; 19 de abril de 2006.

12. Lacerda MR. Tornando-se profissional no contexto domiciliar: vivência do cuidado da enfermeira. [tese] Florianópolis: Universidade Federal de Santa Catarina; 2000.

13. Arrieira ICO, Thofehrn MB, Fripp JC, Duval P, Valadão M, Amestoy SC. Programa de internação domiciliar interdisciplinar oncológico: metodologia de trabalho. Ciência, cuidado e saúde. 2009; supl. 8: 104-9. 
14. Instituto Nacional de Câncer (Brasil). Ações de Enfermagem para o controle do câncer: uma proposta de integração ensino-serviço. Rio de Janeiro: INCA; 2008.

15. Associação Brasileira de Cuidados Paliativos. Consenso Brasileiro de caquexia/Anorexia. Revista Brasileira de Cuidados Paliativos 2011, 3(3); Supl.1.

16. Instituto Nacional de Câncer ((Brasil). Consenso Nacional de Nutrição Oncológica. Rio de Janeiro: INCA; 2009.

17. Ravasco P, Monteiro-Grillo I, Vidal P M, Camilo ME. Nutritional Deterioration in Cancer: The Role of Disease and Diet. Clin Oncol 2003; 5: 443-50.

18. Andrew IM, Waterfield K, Hildreth AJ, Kirkpatrick G, Hawkins C. Quantifying the impact of standardized assessment and symptom management tools on symptoms associated with cancer-induced anorexia cachexia syndrome. Palliat Med 2009; 23(8): 680-8.

19. Ottery FD. Cancer Cachexia: prevention, early diagnosis and management. Cancer Pract 1994; 2 (2):123-31.

20. Marin CCM, et al. Nutritional risk evaluation and establishment of nutritional support in oncology patients according to the protocol of the Spanish nutrition and Cancer Group. Nutrición hospitalaria 2008; 23(5): 458-68.

21. Davis MP, Walsh D, Lagman R, Yavuzsen T. Early satiety in cancer patients: acommon and important but underrecognized symptom. Support Care Cancer 2006; 14(7): 693-8.

22. Monteiro NML, Araujo DF, Basseti SE, Vieira JPFB, Santos MRM, Oliveira Junior PPL, et al. Câncer de Esôfago: perfil das manifestações clínicas, histologia, localização e comportamento metastático em pacientes submetidos a tratamento oncológico em um Centro de Referência em Minas Gerais. Revista brasileira de cancerologia 2009; 55(1): 27-32.

23. Baxter NN, Virnig DJ, Rothenberger DA, Morris AM, Jessurun J, Virnig, BA. Lymph node evaluation in colorectal cancer patients: a population based study. J Natl Cancer Inst 2005; 97(3): 219-25.

24. Silva PB, Lopes M, Trindade Teixeira LC, Yamanouchi $\mathrm{CN}$. Controle dos sintomas e intervenção nutricional. Fatores que interferem na qualidade de vida de pacientes oncológicos em cuidados paliativos. Revista Dor 2010; 11(4): 282-8. 


\section{Abstract}

Introduction: Among gactors associated with malnutrition in cancer, the symptoms related to food intake may contribute significantly to the poor nutritional status. Objective: To assess the prevalence of symptoms related to decreased food intake and its association with tumor location of patients with digestive tract cancer, hospitalized by a Home Care Program for Interdisciplinary Oncology treatment. Method: This is a descriptive study that used secondary data from an Interdisciplinary Oncology Home Care Program. The study included all patients treated for Nutrition, diagnosed with cancer of the digestive tract admitted between February 2006 and February 2011. We analyzed their demographic, clinical and nutritional data. Results: Of the 102 patients studied, whose average age was $61 \pm 14.1$ years old, $100 \%$ were regarded with some degree of malnutrition. Bowel cancer was the most predominant in both sexes, followed by cancer of the esophagus. The most prevalent symptoms corresponded to xerostomia (75.5\%), early satiety $(74.5 \%)$, pain $(60.8 \%)$ and anorexia $(53.9 \%)$. The prevalence of dysphagia in patients with tumor of the esophagus was approximately four times greater when compared to patients with other tumors of the digestive system $(\mathrm{p}<0.01$ IC 95\% 2.35 - 6.48). Conclusion: Xerostomia, early satiety, pain, and anorexia may have contributed to lower food intake, which increases the nutritional status of these patients. Through this knowledge nutritional strategies can be developed to prevent or improve the condition.

Key words: Digestive System Neoplasms; Eating; Symptoms; Malnutrition; Cross-Sectional Studies; Epidemiology, Descriptive

\section{Resumen}

Introducción: Entre los factores asociados con la desnutrición en el cáncer, los síntomas relacionados con la ingesta de alimentos pueden contribuir de manera significativa con el comprometimiento del estado nutricional. Objetivo: Evaluar la prevalencia de los síntomas relacionados con la reducción de la ingesta de alimentos y su asociación con la localización del tumor, de pacientes con cáncer del tracto digestivo, ingresados en un Programa de Internación Domiciliaria Interdisciplinaria de Oncología. Método: Estudio descriptivo que utilizó datos secundarios de un Programa de Internación Domiciliaria Interdisciplinar Oncológico. Participaron en el estudio todos los pacientes tratados por el Servicio de Nutrición, con diagnóstico de cáncer del tracto digestivo que ingresaron en el programa en el periodo de febrero de 2006 hasta febrero de 2011, y han sido analizados sus datos demográficos, clínicos y nutricionales. Resultados: De los 102 pacientes estudiados, con una edad promedia de $61 \pm 14,1$ años, el 100\% han sido considerados con algún grado de desnutrición. El cáncer de colon ha sido el más predominante en ambos sexos, seguido por el cáncer del esófago. Los síntomas más prevalentes correspondieron al xerostomía $(75,5 \%)$, saciedad precoz $(74,5 \%)$, dolor $(60,8 \%)$ y anorexia $(53,9 \%)$. La prevalencia de la disfagia en pacientes con tumor de esófago fue aproximadamente cuatro veces mayor en comparación con pacientes con otros tumores del aparato digestivo ( $\mathrm{p}<0,01$ IC 95\%: 2,35 a 6,48). Conclusión: Xerostomía, saciedad precoz, dolor y la anorexia que puede haber contribuido a la menor ingesta de alimentos, lo que agrava el estado nutricional de estos pacientes. A través de ese conocimiento las estrategias nutricionales podrán ser desarrolladas para prevenir y, o mejorar esta condición.

Palabras clave: Neoplasias del Sistema Digestivo; Ingestión de Alimentos; Desnutrición; Sintomas; Estudios Transversales; Epidemiología Descriptiva 